\title{
A Role of YlBud8 in the Regulation of Cell Separation in the Yeast Yarrowia lipolytica
}

\author{
Yun-Qing Li* , Qing-Jie Xue, Yuan-Yuan Yang, Hui Wang, and Xiu-Zhen Li \\ Department of Pathogenic Biology, Jining Medical University, Shandong 272067, China
}

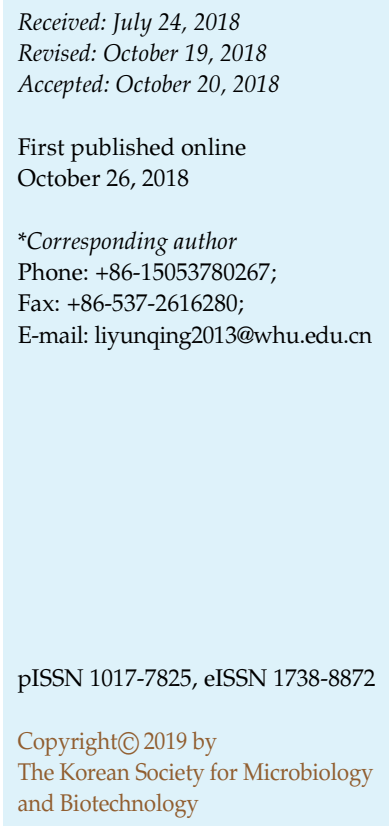

The spatial landmark protein Bud8 plays a crucial role in bipolar budding in the budding yeast Saccharomyces cerevisiae. The unconventional yeast Yarrowia lipolytica can also bud in a bipolar pattern, but is evolutionarily distant from $S$. cerevisiae. It encodes the protein YALI0F12738p, which shares the highest amino acid sequence homology with S. cerevisiae Bud8, sharing a conserved transmembrane domain at the C-terminus. Therefore, we named it YlBud8. Deletion of YlBud8 in Y. lipolytica causes cellular separation defects, resulting in budded cells remaining linked with one another as cell chains or multiple buds from a single cell, which suggests that YlBud8 may play an important role in cell separation, which is distinct from the function of Bud8 in S. cerevisiae. We also show that the YlBud8-GFP fusion protein is located at the cell membrane and enriched in the bud cortex, which would be consistent with a role in the regulation of cell separation. The coiled-coil domain at the Nterminus of YlBud8 is important to the correct localization and function of YlBud8, as truncated proteins that do not contain the coiled-coil domain cannot rescue the defects observed in Ylbud8s. This finding suggests that a new signaling pathway controlled by YlBud8 via regulation of cell separation may exist in Y. lipolytica.

Keywords: YlBud8, cell separation, cell polarity, coiled-coil

\section{Introduction}

Cell polarization is central to the development of most organisms as it plays important roles in cell differentiation, division, cell-cell signaling, and cell migration [1]. Many cellular physiological activities are directional, including nutrient transport, nerve signal transmission, as well as cell movement, and cell polarity is necessary for the normal function of these and other cellular activities. Saccharomyces cerevisiae is a classic model organism. Its cells display significant polarity when sensing and responding to external or internal signals, and polar growth is involved in many steps of the cell cycle, including budding site selection, bud growth, mating, mycelial growth and cell separation [2, 3]. Cell separation is important for both increased cell number and cell differentiation, and is considered to be a special form of polar growth [4]. In budding yeast, both budding and cell separation require actin polarization and exocytosis. During the process of budding, polar growth is directed to the cortex of the bud to assemble a new bud, while at the later stage of the cell cycle, polar growth is directed to the bud neck to facilitate cytokinesis [5, 6]. Site selection in budding and cell division is nonrandom, but rather is controlled by spatial signals [7]. Thus, it is important to understand how spatial cues determine sites of polarized cell growth.

The molecular regulation of this process has been thoroughly studied in S. cerevisiae. A large number of spatial landmarks that are involved in polarized cell growth during bud site selection have been identified, which exist in and are expressed in different cell types. There are two sets of landmark cues that can mark new bud sites. In haploid cells, the polar sites are marked by the axial landmarks Axl1, Axl2, Bud3, and Bud4. In diploid cells, the polar sites are marked by the bipolar landmarks Rax1, Rax2, Bud8, and Bud9 [8, 9]. The bud-site GTPase Rsr1 and its regulators, the GTPase activating protein (GAP) Bud2 and the guanine nucleotide exchange factor 
(GEF) Bud5 make up the core module, which can recognize the positional cues that mark the poles of the haploid and diploid cells [10]. Then, the Rsr1 GTPase module can regulate the GTPase Cdc42, which associates with effector proteins to establish cell polarity, initiate polarized growth, and assemble the new bud at specific sites [11-13].

Spatial control of polar growth is not a phenomenon unique to $S$. cerevisiae, but exists ubiquitously in other yeasts. For example, the non-model yeast Yarrowia lipolytica buds in a bipolar pattern: mother cells can choose the bud site at either the proximal or distal poles to the preceding site of cytokinesis, whereas the daughter cells usually bud at the distal pole to the birth site, this process is thought to be controlled by spatial cues [14]. With Y. lipolytica, the bipolar bud takes on a very steady budding pattern that exists without the influence of cell type change, cell morphology change, or changes in the external environment; this characteristic is different from those of Candida albicans and $S$. cerevisiae $[15,16]$. We are interested in the spatial landmarks that are involved in regulating the stable budding pattern of $Y$. lipolytica, a hemiascomycetous yeast species distantly related to the model organism S. cerevisiae. This species exhibits several special characteristics at the physiological, biochemical and metabolic levels and has recently become a model organism as unconventional yeast [17].

Research on the spatial landmark for bipolar budding in $Y$. lipolytica appears to be thus far absent. In S. cerevisiae, Bud8 and Bud9 are thought to act as bipolar spatial landmarks that provide spatial signals in diploid cells [18, 19]. Bud8 is a part of the distal landmark, which is required for distal bud site selection; Bud9 is localized to the proximal pole and is required for proximal pole selection
[20]. These two proteins can interact with Bud5 to deliver the landmark signals to the Rsr1 GTPase module [9]. We had previously discovered that YlRsr1 is involved in the regulation of the bipolar budding pattern and cell separation in Y. lipolytica. Cells without YlRsr1 cannot separate from one another and form cell chains or multibuds [21]. To understand whether spatial landmarks similar to Bud8 and Bud9 exist to provide spatial signals to the YlRsr1 GTPase module in Y. lipolytica, the amino acid sequences of Bud8 and Bud9 from S. cerevisiae were used in a BLAST search for homologue proteins in the NCBI database (https:/ / www.ncbi.nlm.nih.gov/) where the optional organism is $Y$. lipolytica.

Here, we show that the Bud8 homologue YlBud8 plays a crucial role in cell separation, but is not involved in regulating bipolar budding. A YlBud8-GFP fusion protein can be located at the cell membrane and the small bud cortex, which is different from where S. cerevisiae Bud8 is located. We further show that the coiled-coil domain in YlBud8 is required for its normal function and localization.

\section{Materials and Methods}

\section{Strains, Media and Growth Conditions}

The Y. lipolytica strains used in this study are listed in Table 1. The construction of strains and strain source are described below or in Table 1. Escherichia coli strain DH5 $\alpha$ was used for plasmids amplification. Y. lipolytica strains were grown in YPD medium ( $1 \%$ yeast extract, $2 \%$ peptone, and $2 \%$ glucose) or in synthetic YNBD medium $(0.67 \%$ yeast nitrogen base without amino acid, $2 \%$ glucose) at $30^{\circ} \mathrm{C}$. YNBD media were supplemented with $20 \mathrm{mg} / \mathrm{l}$ uracil, $80 \mathrm{mg} / \mathrm{l}$ leucine, or both when required. Agar was added to $2 \%$ concentration for solid media, and glucose was used in place of glycerin to keep cells in their oval form in synthetic media.

Table 1. Y. lipolytica strains used in this study.

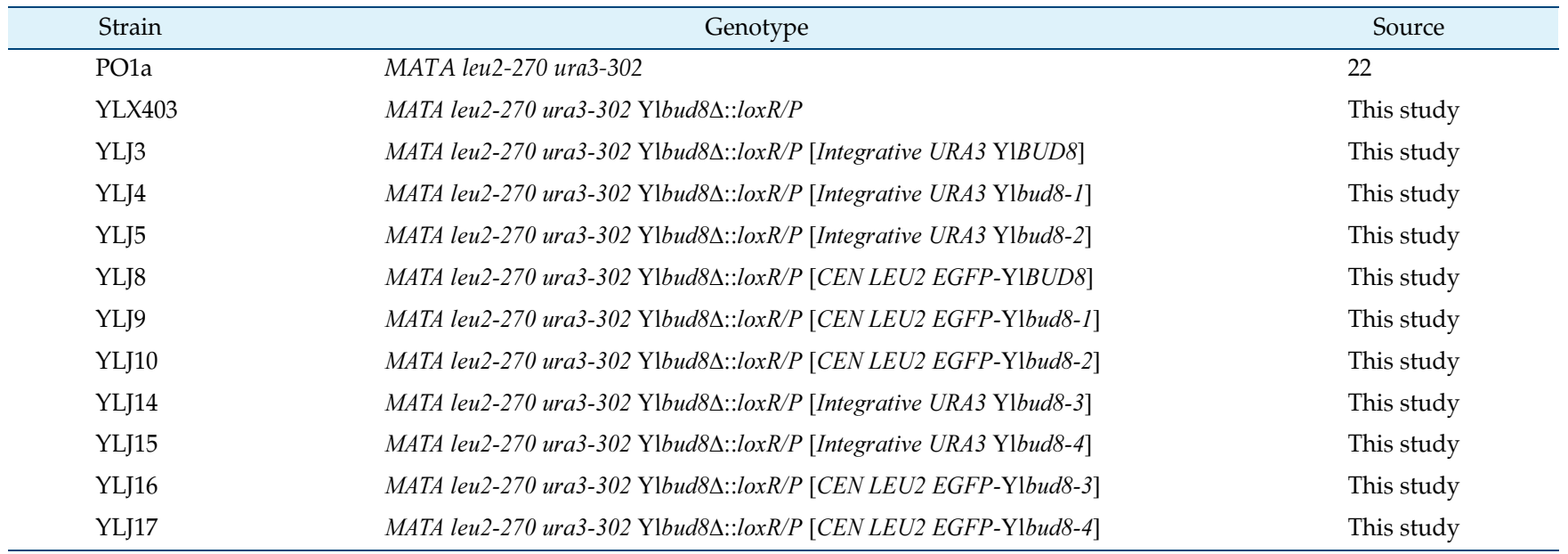


Table 2. Plasmids used in this study.

\begin{tabular}{|c|c|c|}
\hline Plasmid & Description & Source \\
\hline pRRQ2 & CEN Y1LEU2 hp4d-CRE & Jean-Marc Nicaud \\
\hline pWU24 & YIURA3 (178 bp promoter, ORF and 772 bp 3' UTR) in pBlueScript KS(+) & 23 \\
\hline pWU24-Y1BUD8 & YlBUD8 in pWU24 & This study \\
\hline pWU25 & $P_{\text {YвUD } 8}(680$ bp promoter $)$ in pWU24 & This study \\
\hline pWU25-Ylbud8-1 & Ylbud8-1 in pWU25 & This study \\
\hline pWU25-Ylbud8-2 & Ylbud8-2 in pWU25 & This study \\
\hline pWU25-Ylbud8-3 & Ylbud8-3 in pWU25 & This study \\
\hline pWU25-Ylbud8-4 & Ylbud8-4 in pWU25 & This study \\
\hline pYL8 & loxR-YIURA3-loxP Y1LEU2 in pBlueScript KS(+) & 23 \\
\hline pYL8-YlBUD8-PT & $P_{\text {YвиD } 8}-l o x R-Y 1 U R A 3-l o x P-T_{\text {YвUD } 8}$ in pYL8 & This study \\
\hline pYL15 & $P_{\mathrm{YITEF1}}-E G F P$ in pINA445 & 24 \\
\hline pYL15-YlBUD8 & $P_{\mathrm{YITEF} 1}-E G F P-\mathrm{Y} 1 B U D 8$ in pYL15 & This study \\
\hline pYL15-Ylbud8-1 & $P_{\text {YITEF1 }}-E G F P-Y 1 b u d 8-1$ in pYL15 & This study \\
\hline pYL15-Ylbud8-2 & $P_{\mathrm{YITEF1}}-E G F P-Y 1 b u d 8-2$ in pYL15 & This study \\
\hline pYL15-Ylbud8-3 & $P_{\mathrm{YITEF}}-E G F P-Y 1 b u d 8-3$ in pYL15 & This study \\
\hline pYL15-Ylbud8-4 & $P_{\mathrm{YITEF1}}-E G F P-Y 1 b u d 8-4$ in pYL15 & This study \\
\hline
\end{tabular}

\section{Construction of Plasmids and Yeast Strains}

Plasmids and primers used in this study are respectively listed in Tables 2 and 3. YlBUD8 was deleted in the wild-type strain PO1a using homologous recombination, which was used in the deletion of YITEC1 [23]. PCR was used to identify the mutants that carried the correct replacement of YlBud8. Plasmid pRRQ2 was transformed into the YlBUD8 deletion mutant to remove the selection marker YlURA3. We used PCR to confirm the deletion strains with the YlBUD8-5CK \& YlBUD8-1R primers after the YlURA3 marker was removed.

The plasmid pWU24-YlBUD8 was constructed to complement the Ylbud8D mutant. The 4,135-bp YlBUD8 gene containing promoter ( $845 \mathrm{bp}$ ) and a 282-bp 3'-untranslated region (UTR) was digested by ClaI and BamHI and then ligated into pWU24 (integrative, YlURA3) [24]. The plasmids were linearized by PmlI to introduce them into yeast cells for the complementation test.

To generate a version of pWU25 that tests the activity of truncated fragments of YlBud8, the 668-bp KpnI-ClaI YlBUD8 promoter was amplified by PCR and was inserted into pWU24. YlBud8 segments were inserted into the plasmid pWU25 digested by ClaI and BamHI, resulting in pWU25-Ylbud8-1, pWU25Ylbud8-2, pWU25-Ylbud8-3, and pWU25-Ylbud8-4. The plasmids were also linearized, leading to Ylbud8s, which was used to identify the function of the four fragments.

The plasmid pYL15-YlBUD8 was constructed to examine the subcellular localization of YlBud8. To do this, the 3,280-bp YlBUD8 gene containing the full ORF and a 282-bp 3'-UTR sequence was amplified by PCR from genomic DNA and ligated into XbaI- and BamHI-digested pYL15 (CEN, YlLEU2, $P_{\text {YITEF } 1}$-EGFP) [27], the Nterminus of YlBud8 was fused with EGFP. The truncated fragments were also amplified from genomic DNA and inserted into the XbaI- and BamHI-digested pYL15. The five plasmids were all introduced into Ylbud8s cells to examine the differences in localization of the different truncated fragments.

\section{Yeast Transformation}

The lithium acetate method was used to introduce plasmids to Y. lipolytica strains. This method has been used in the transformation of S. cerevisiae, but with Y. lipolytica, the cells were heat shocked at a lower temperature for a shorter period of time before plating on selective medium: $37^{\circ} \mathrm{C}$ for $15 \mathrm{~min}$.

\section{Western-Blot Analysis of Proteins}

Cells of strain Ylbud8 $\Delta$ carrying pYL15 or pYL15-YlBud8 segments were grown in YNBL+Ura medium at $30^{\circ} \mathrm{C}$ for $16 \mathrm{~h}$. To extract total cellular proteins the Yeast Protein Extraction Reagent (Takara, Japan) was used. Proteins were separated by 8.0\% SDSPAGE. Mouse monoclonal antibody against GFP (Abcam, England) was used as primary antibody and horseradish peroxidaseconjugated goat anti-mouse IgG was used as secondary antibody.

\section{Microscopy}

Cell morphology was observed using an Olympus BX51 microscope (Japan) and a DP80 charge-coupled-device (CCD) camera. The images were acquired using CellSens Standard. For the count of the percentage of cells with abnormal morphology, a minimum of 200 cells were counted.

\section{Results}

\section{Identification of YlBud8 in Y. lipolytica}

In order to find the potential bipolar landmark in Y. lipolytica, the amino acid sequences of Bud8 and Bud9 from 
Table 3. Primers used in this study.

\begin{tabular}{|c|c|c|}
\hline Name & Sequence $\left(5^{\prime} \rightarrow 3^{\prime}\right)$ & Use \\
\hline YlBUD8-PF & CGCGGATCCGAGATATAGCGTATGGTCAGC & Amplification of $\mathrm{P}_{\mathrm{Y} \mid B U D 8}$ and $\mathrm{Y} 1 B U D 8$ \\
\hline YlBUD8-PR & CCGGAATTCAGAAGTTGTAGTGAAGTAGCG & Amplification of $\mathrm{P}_{\text {YІBUD8 }}$ \\
\hline YlBUD8-TF & ACCCAAGCTTTGGTTGCTGGCGTTTTAACGA & Amplification of $\mathrm{T}_{\text {YIBUD } 8}$ \\
\hline YlBUD8-TR & CCCAGCGTCGACTAGGAGTCTAGCCAAACGACC & Amplification of $\mathrm{T}_{\text {YाBUD8 }}$ \\
\hline YlBUD8-5CK & ACAACAGATGAAGAAGAG & Checking Ylbud8 $\Delta$ deletion \\
\hline YlBUD8-1PF & CGGGGTACCGACATGAAATCTCATGGCTTCAG & Amplification of $\mathrm{P}_{\text {YвВLD8 }}$ \\
\hline YlBUD8-1PR & CCCATCGATAGAAGAAGTTGTAGTGAAGTAG & Amplification of $\mathrm{P}_{\text {YвUD } 8}$ \\
\hline YlBUD8-1R & ACATGCATGCCGCTTTGTCAGACTGTCTACC & Checking Ylbud8 $\Delta$ deletion \\
\hline YlBUD8-1F & CCCATCGATTGCACGAACTCCACATTCCACC & Amplification of YlBUD8 \\
\hline YlBUD8-2R & CGCGGATCCAATGCTCAGTAATGACTGATGG & Amplification of YlBUD8, Ylbud8-1, Ylbud8-2 \\
\hline YlBUD8-2F & CCCATCGATATGCTCACCGACATTAACAACGA & Amplification of Ylbud8-1 \\
\hline YlBUD8-3F & CCCATCGATATGGTGGATAATATTGTCACAG & Amplification of Ylbud8-2 \\
\hline YlBUD8-7F & CCCATCGATATGAATGTCTCTGTTGACTCCTT & Amplification of Ylbud8-3 \\
\hline YlBUD8-8F & CCCATCGATATGGGCGAGGGTGAGGATGAG & Amplification of Ylbud8-4 \\
\hline YlBUD8-4F & TGCTCTAGAATGGACACGAAACGAAGCCTTTC & Amplification of YlBUD8 \\
\hline YlBUD8-5F & TGCTCTAGACTCACCGACATTAACAACGAG & Amplification of Ylbud8-1 \\
\hline YlBUD8-6F & TGCICTAGAGTGGATAATATTGTCACAGTC & Amplification of Ylbud8-2 \\
\hline YlBUD8-9F & TGCTCTAGAAATGTCTCTGTTGACTCCTT & Amplification of Ylbud8-3 \\
\hline YlBUD8-10F & TGCTCTAGAGGCGAGGGTGAGGATGAG & Amplification of Ylbud8-4 \\
\hline
\end{tabular}

S. cerevisiae were used in a BLAST search and compared against the NCBI database (https:/ / www.ncbi.nlm.nih.gov/) to find proteins that share the highest degree of similarity in amino acid sequences. The protein encoded by the ORF YALI0F12738 satisfied the above condition, sharing $11 \%$ identity with Bud8 and 10\% identity with Bud9 from S. cerevisiae. Although these are not considered to be high degrees of homology, YALI0F12738 was the sequence sharing the highest degree of homology among Y. lipolytica proteins. We then analyzed the conserved domains of these three proteins use SMART (http://smart.embl-heidelberg.de/) and discovered that YALIOF12738p shares the same conserved transmembrane (TM) domain at its C-terminus and that this domain shares $23 \%$ and $22 \%$ amino acid sequence identity with the TM domains of Bud8 and Bud9, respectively (Fig. 1). In addition, we found that YALIOF12738 is 995 amino acids in length with a long N-terminus containing a coiled-coil domain. In comparison, Bud8 and Bud9 encode a 603-amino acid protein and a 547-amino acid protein, respectively, with a shorter $\mathrm{N}$-terminus and without a coiled-coil domain, which may be the reason that the degree of homology between these proteins is so low. We named the Y. lipolytica homolog protein YlBud8 and analyzed its function.

\section{YlBud8 Plays an Important Role in Cell Separation}

S. cerevisiae cells lacking either Bud8 or Bud9 are normal in cell morphology and growth, with changes observed only in bipolar budding. To determine whether YlBud8 is required for bipolar budding, homologous recombination

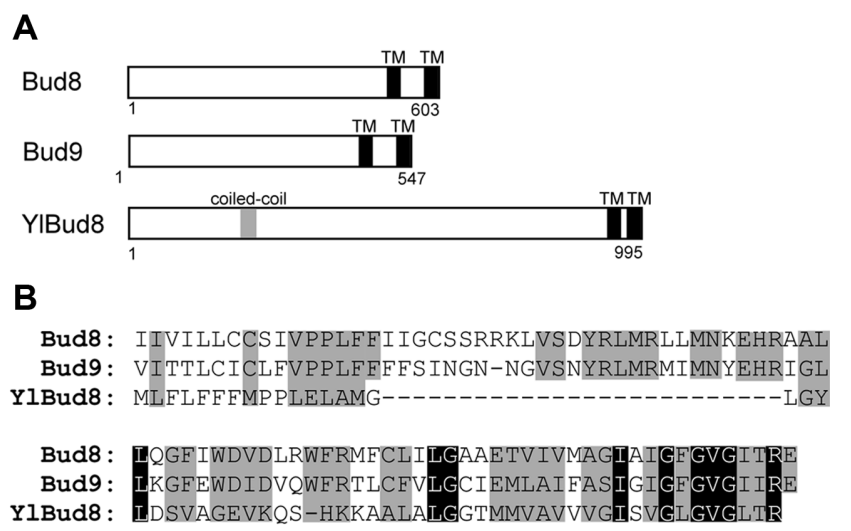

Fig. 1. Homology domains in Bud8 and Bud9.

(A) Map of S. cerevisiae Bud8, Bud9 and Y. lipolytica YlBud8 domains, TM domain is shown in black and the coiled-coil domain is shown in gray. (B) Sequence alignment of the TM domains in S. cerevisiae Bud8, Bud9 and YlBud8. Black shading: identical residues; gray shading: similar residues. 

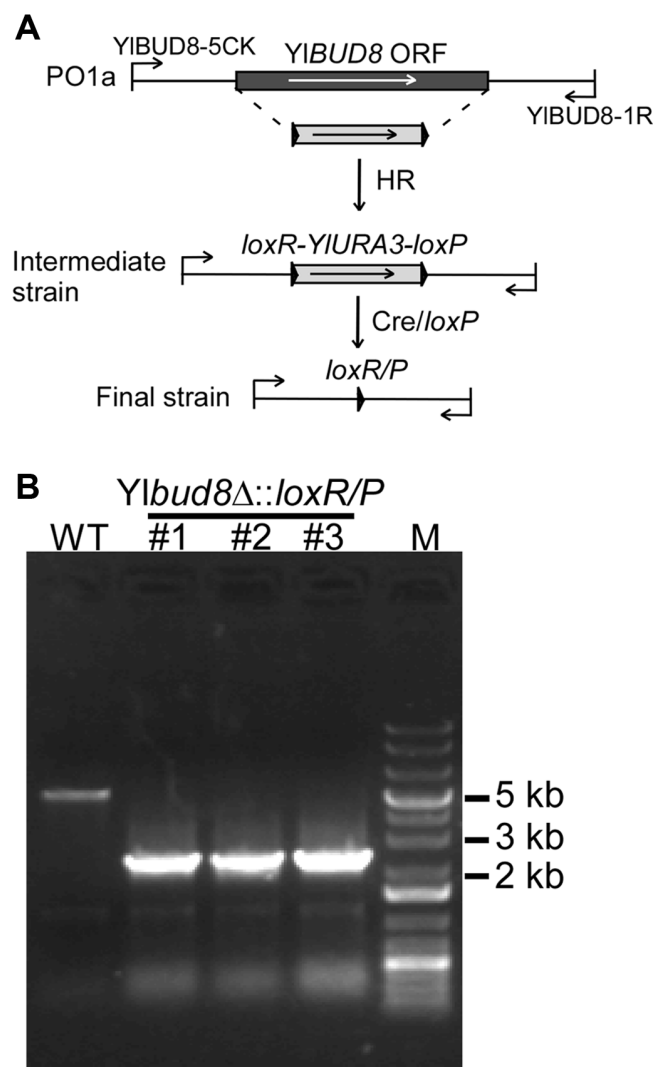

Fig. 2. Verification of Y. lipolytica gene deletion mutant.

(A) Schematic representation of YlBUD8 deletion procedure. The YlBUD8 ORF was first replaced by the loxR-YlURA3-loxP marker via homologous recombination. Then, the YlURA3 marker was removed. The location of the primers YlBud8-5CK \& YlBud8-1R used for PCR is shown. (B) Verification of YlBUD8 deletion in three independent clones of the strain Ylbud8 $\Delta:: l o x R / P$. The primer pair YlBUD8-5CK/ YlBUD8-1R was used to amplify the YlBUD8 locus in strains PO1a (WT) and Ylbud8s::loxR/P. Expected amplified DNA fragments are $5.1 \mathrm{~kb}(\mathrm{WT})$ and $2.1 \mathrm{~kb}(\mathrm{Ylbud} 8 \Delta::$ loxR/P).

was used to delete YlBUD8 from the Y. lipilytica wild-type strain PO1a. We successfully obtained three mutants where Y1BUD8 was correctly deleted, and the deletion was verified by PCR. When the wild-type genome was used as a template, we were able to obtain a PCR product of $5.1 \mathrm{~kb}$, while a PCR product of $2.1 \mathrm{~kb}$ was obtained when the mutant genomes were used as the PCR template (Fig. 2), which indicated that YlBUD8 was correctly deleted. We then named the mutant YLX403 and observed its phenotype to help identify the function of YlBud8.

In YPD liquid medium, cells that are Ylbud8s exhibit bipolar budding, which is the same as observed in the wild-type PO1a (data not shown). This result suggests that YlBud8 is not involved in the regulation of bipolar budding in Y. lipolytica. In order to find the function of YlBud8, we examined Ylbud8s cells in yeast form and during filamentous growth. We did not observe a detectable growth defect in Ylbud8s cells at $30^{\circ} \mathrm{C}$ (data not shown), but cells showed morphological defects during yeast form and filamentous growth. In liquid YPD medium (yeast form), most Ylbud8 $(80 \%, n=200)$ cells remain linked to one another and form cell chains or multiple buds, while the wild-type PO1a forms single and oval form cells, and only $10 \%(n=200)$ of the cells form cell chains (Figs. $3 \mathrm{~A}$ and 3B). We also examined the cell morphology in YNBD and YNDC7 media that can promote mycelial growth to detect their ability to form pseudohyphae and hyphae. The PO1a strain formed an elongated morphology in YNBD medium and a few hyphae in YNDC7 medium. In YNBD medium, more

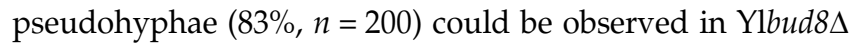
cells than in wild-type cells $(7 \%, n=201)$ (Fig. 3C). The same phenomenon was observed when cells were grown in YNDC7 medium, which can induce hyphae growth, as the majority of cells $(85 \%, n=200)$ formed pseudohyphae where the length of a single cell is the same as that of the wild-type cell (Fig. 3D). When the intact plasmid pWU24YlBUD8 was used for the complementation of the Ylbud8s strain, the cell morphology recovered well. This result proves that the defects we observed are caused by the deletion of Y1BUD8 and that Y1BUD8 was correctly deleted.

Together, we conclude that YlBud8 is not involved in the regulation of bipolar budding but plays an important role in cell separation of Y. lipolytica during cell growth.

\section{The Coiled-Coil Domain in YlBud8 is Important for Regulating Cell Separation}

YlBud8 has a long N-terminus with a coiled-coil domain which is absent in Bud8 and Bud9 from S. cerevisiae. The coiled-coil domain is a common structural pattern that mediates protein-protein interactions [25]. Therefore, we wanted to find out whether the long $\mathrm{N}$-terminus or the coiled-coil domain plays an important role in the normal function of YlBud8. We constructed four truncated constructs of YlBud8, named Ylbud8-1, Ylbud8-2, Ylbud8-3 and Ylbud84. The long N-terminus of YlBud8 was removed but the coiled-coil domain was retained in Ylbud8-1 and Ylbud8-3 while in Ylbud8-2 and Ylbud8-4 the long N-terminus and the coiled-coil domain were both removed (Fig. 4A). The truncated Ylbud8 segments were expressed in Ylbud8 $\Delta$ cells under the control of the YlBud8 promoter to determine whether they can rescue the defects in cell morphology found in Ylbud8s. We examined the cell morphology of Ylbud8s cells expressing YlBud8 segments in YPD liquid. 
A

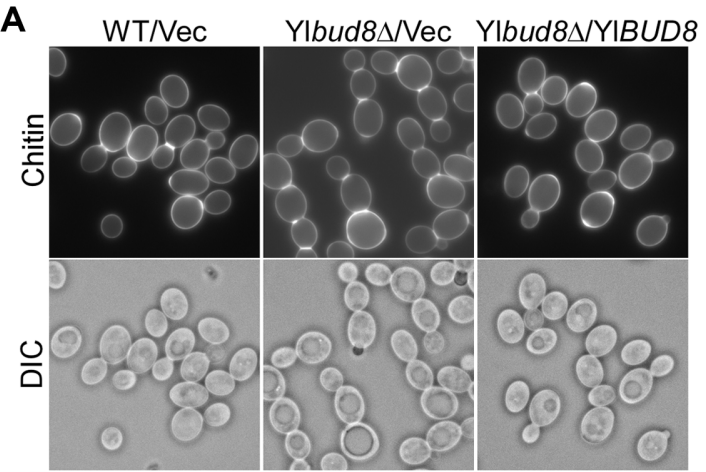

C

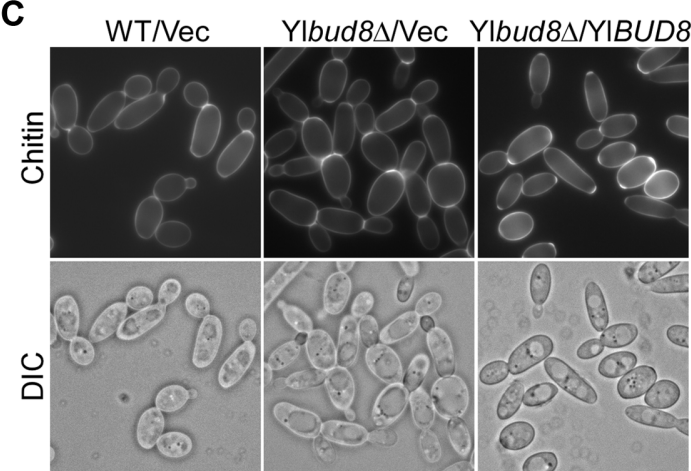

B

\begin{tabular}{|c|c|c|}
\hline Strains & Single cells $(\%)$ & Cell chains $(\%)$ \\
\hline WT/Vec & 90 & 10 \\
\hline Ylbud8s/Vec & 18 & 82 \\
\hline Ylbud8A/YIBUD8 & 88 & 12 \\
\hline
\end{tabular}

D

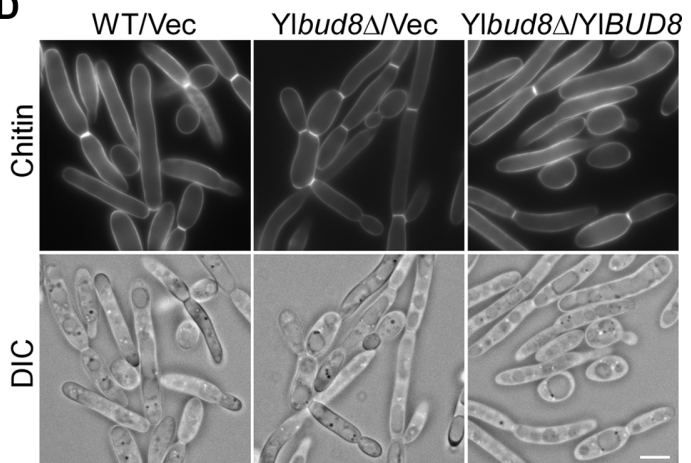

Fig. 3. Phenotypes of Ylbud8s cells.

(A) Cells of PO1a strain with plasmid pWU24 (WT/Vec) and YLX403 strain with plasmid pWU24 (Ylbud8s/Vec) or pWU24-Y1BUD8 (Ylbud84/ $\mathrm{YlBUD8}$ ) were grown in liquid YPD medium at $30^{\circ} \mathrm{C}$ for $12 \mathrm{~h}$, then were stained with Calcofluor white. (B) The percentage of single cells and cell chains were counted. (C and D) The same cells shown in A were grown in liquid YNBD and YNDC7 media at $30^{\circ} \mathrm{C}$ for $16 \mathrm{~h}$. Scale bar, $5 \mu \mathrm{m}$.

We found that YlBud8, Ylbud8-1, and Ylbud8-3 can rescue the cell morphology defect of Ylbud8s cells during yeast form growth (Fig. 4C). In YPD medium, $83 \%(n=200)$ of

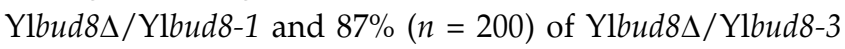
cells show normal cell shape and size. Only $17 \%$ or $13 \%$ of cells are connected with one another in short cell chains or multiple buds, which is close to the $8 \%(n=221)$ short cell chains or multiple buds found in Ylbud8s/YlBud8 cells (Fig. 4B). These results suggest that the long N-terminus deletion in YlBud8 is largely functional in cell separation and further investigation will be needed to elucidate the function of the coiled-coil domain.

The truncated Ylbud8-2 and Ylbud8-4 segments were expressed in Ylbud8D cells under the control of the YlBUD8 promoter to study whether they can rescue the defect of Ylbud8s in cell morphology. Our results show that neither Ylbud8-2 nor Ylbud8-4 can rescue the cell morphology defect of Ylbud8s during yeast form growth. When grown in YPD medium, $75 \%(n=200)$ of Ylbud8s/Ylbud8-2 and $79 \%(n=200)$ of Ylbud8 $\Delta /$ Ylbud8-4 cells are linked together with one another and form cell chains or multiple buds, which is similar to the phenotype of Ylbud8s mutants (Fig. 4B and 4C). Therefore, we conclude that the coiledcoil domain is essential for the function of YlBud8 to regulate cell separation, but the long $\mathrm{N}$-terminus is not required for this purpose.

\section{The Coiled-Coil Domain Plays an Important Role in the Localization of Y1Bud8}

Protein localization is often linked to function. To obtain more information about the function of YlBud8, we fused EGFP to the N-terminus of YlBud8 to observe the location of EGFP-YlBud8. The fusion construct EGFP-YlBUD8 was expressed using the YITEF1 promoter and was fully functional, as it rescued the cell morphology defect of Ylbud8s. The fusion protein can locate to the plasma membrane uniformly in unbudded cells, and is enriched in the bud cortex in both small-budded and large-budded cells (Fig. 5A). This result is consistent with the finding that YlBud8 may play an important role in cell separation. 
A

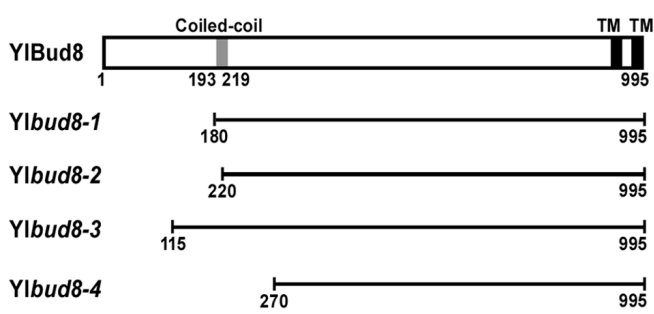

B

\begin{tabular}{ccc}
\hline Strains & Single cells (\%) & Cell chain (\%) \\
\hline YlBud8 & 90 & 10 \\
Ylbud8-1 & 85 & 15 \\
Ylbud8-2 & 25 & 75 \\
Ylbud8-3 & 87 & 13 \\
Ylbud8-4 & 21 & 79 \\
\hline
\end{tabular}
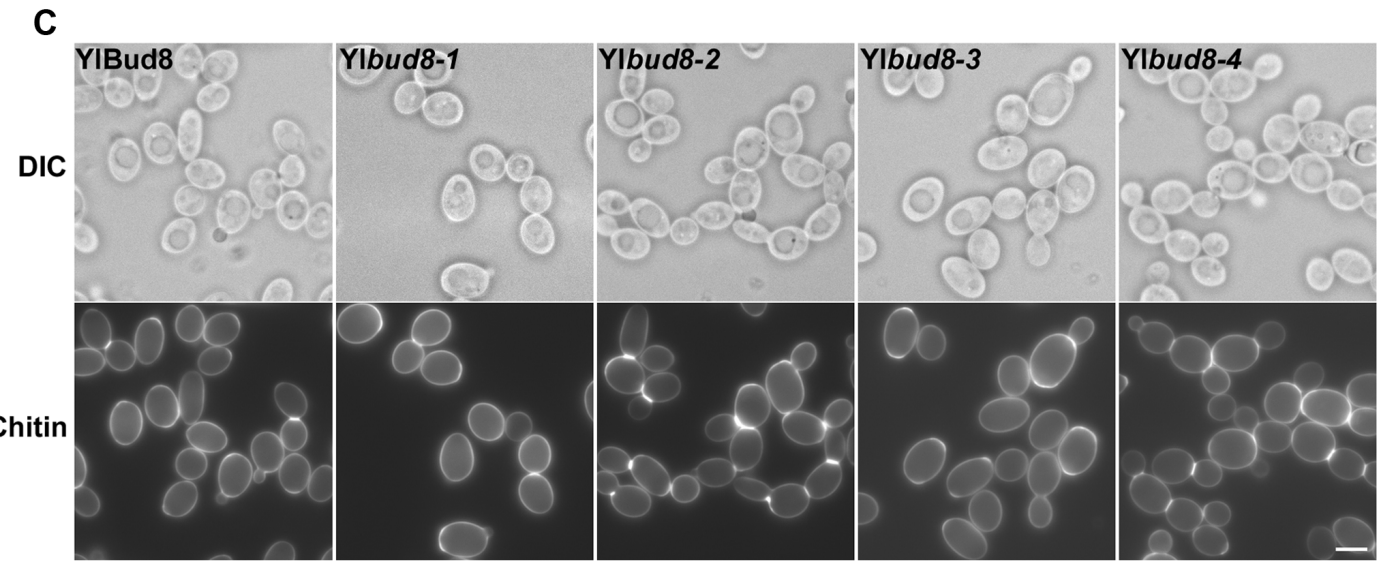

Fig. 4. The coiled-coil domain in YlBud8 plays an important role in regulating cell separation.

(A) Schematic representation of the domains of YlBud8 and the position of YlBud8 truncated segments Ylbud8-1, 2, 3, 4. (B) Cells of strain Ylbud8 integrated with PmlI-linearized plasmids pWU24-YlBud8 (YlBud8) and pWU25-Ylbud8 fragments were grown in liquid YPD medium for $12 \mathrm{~h}$, and the percentage of single cells and cell chains was determined. (C) Same cells in B were stained with calcofluor white. Scale bar, $5 \mu \mathrm{m}$.

We also fused the truncated fragments of YlBud8 to EGFP at their N-terminus to study their localization. YlBud8 and the truncated fragments were all expressed correctly and stably as determined by immunoblotting with a monoclonal antibody against GFP (Fig. 5B). The functions of the fusion proteins are similar to the proteins that were expressed at the basal level in the pWU25 plasmid in the previous section. Fragments EGFP-Ylbud8-1 and EGFP-Ylbud8-3 localize to the same locations as EGFPYlBud8, as they can be observed in the plasma membrane and the bud cortex. However, the fragments EGFP-Ylbud82 and EGFP-Ylbud8-4, which cannot rescue the cell separation defect of Ylbud8s, cannot locate to the cell membrane and bud cortex, but rather remain dispersed in the cytoplasm (Fig. 5A). The above results indicate that the localization of YlBud8 to the cell membrane and the bud cortex are essential to its function in cell separation, and that the mutants Ylbud8-2 and Ylbud8-4, which do not contain the coiled-coil domain, are incorrectly localized. This finding indicates that the coiled-coil domain is important to the localization of YlBud8, and that correct localization of YlBud8 may be a functional determinant.

\section{Discussion}

\section{YlBud8 Plays Important Roles in Cell Separation}

In S. cerevisiae, Bud8 and Bud9 are the essential components of the bipolar landmarks in diploid strains. The deletion of either Bud8 or Bud9 can change the bipolar budding pattern of the diploid strain, but has no influence on cell morphology and cell separation [26, 27]. We have identified YlBud8 in Y. lipolytica as a homolog of Bud8 and Bud9 and studied its function. The data we present above show that YlBud8 plays an important role in cell separation, as YlBud8 deletion mutants cannot separate from one another and ultimately form cell chains or multi-bud cells. The program TMHMM server v.2.0 (http:/ /www.cbs.dtu.dk/ services/TMHMM-2.0) predicts that there are two short TM domains at the C-terminus of YlBud8 and we speculate that the N-terminus of YlBud8 may be located to the extracytoplasmic space, which can provide a spatial basis for YlBud8 to interact with other proteins that can regulate cell separation. In Y. lipolytica, deletion of YlRsr1 can also result in the formation of cell chains or multi-bud cells, which is similar to the phenotypes observed with YlBud8 

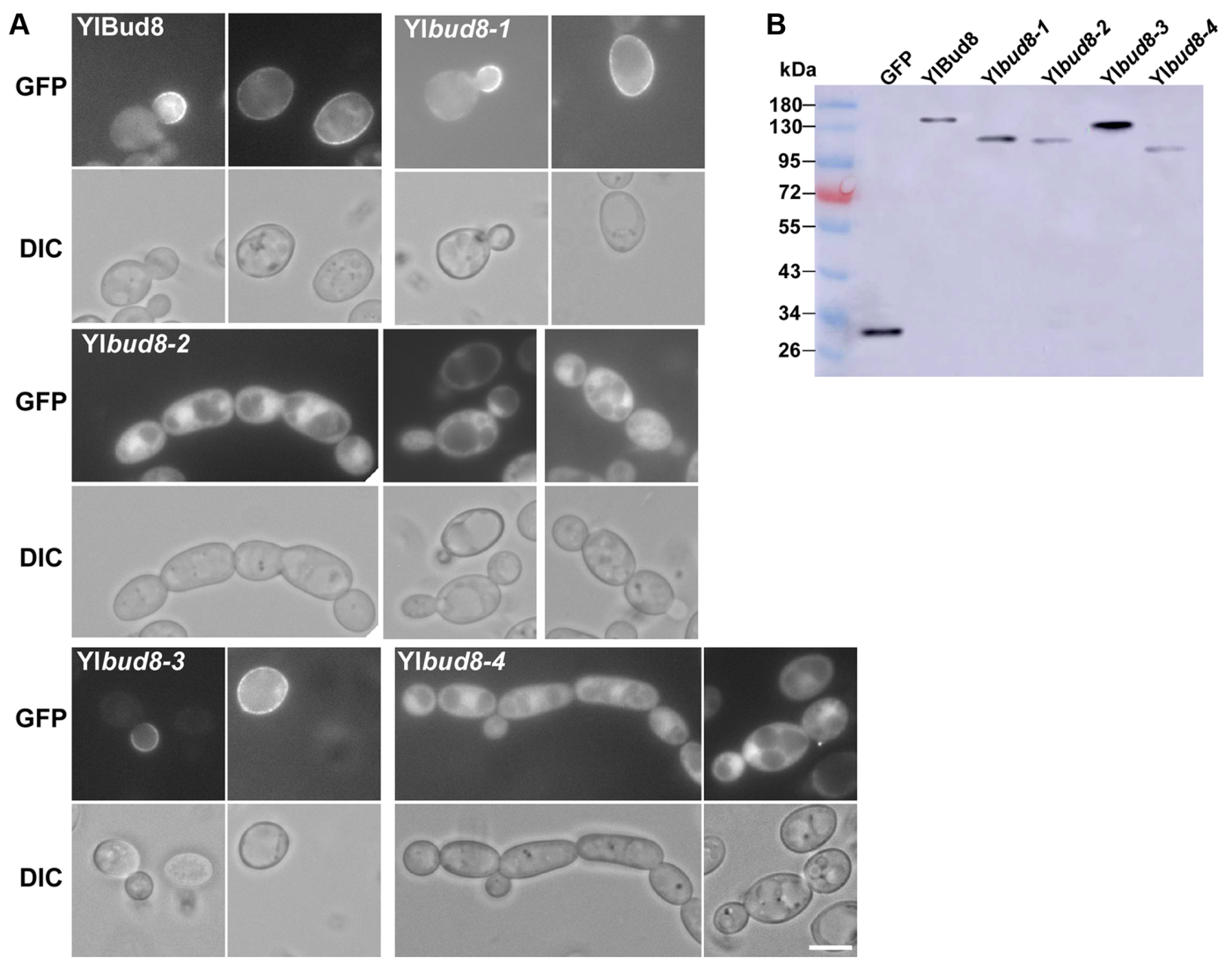

Fig. 5. The coiled-coil domain plays an important role in the localization of YlBud8.

(A) Cells of strain YLX403 (Ylbud8s) carrying plasmids pYL15-YlBud8 fragments were grown in liquid YNBL medium supplemented with uracil at $30^{\circ} \mathrm{C}$ to observe GFP fluorescence. Scale bar, $5 \mu \mathrm{m}$. (B) Yeast strains in A were grown in liquid YNBL+Ura medium for $12 \mathrm{~h}$ and total proteins were extracted, which were separately subjected to $8.0 \%$ SDS-PAGE and immunoblotted with an anti-GFP antibody.

deletion cells [21]. We speculate that YlBud8 may act as the spatial landmark for cell separation and transmit spatial signals to YlRsr1, so that the function of YlBud8 in cell separation may be mediated by the Bud $8 \rightarrow \mathrm{Rsr} 1 \rightarrow \mathrm{Cdc} 24 \rightarrow$ Cdc42 signaling pathway.

We propose two reasons for the differences between the functions of the two homologous proteins Bud8 and YlBud8. Firstly, the structure of YlBud8 differs significantly from that of Bud8 as it has a long $\mathrm{N}$-terminus and a coiledcoil domain at the N-terminus which is non-existent in S. cerevisiae Bud8. Coiled-coils are known as domains that can mediate protein-protein interactions [25]. We speculate that the coiled-coil domain endows YlBud8 with novel functions that may influence the roles it plays in Y. lipolytica. YlBud8 may interact with other proteins through the coiled-coil domain to take part in cell separation, but not in bipolar budding. Secondly, the location of YlBud8 differs from that of Bud8 in S. cerevisae. In S. cerevisiae, Bud8 can localize to the bud tips and presumptive bud sites, which provides the spatial basis for the role played by Bud8 in the regulation of bipolar budding [26]. However, in Y. lipolytica, YlBud8 localizes to the cell membrane and the small bud cortex, but cannot be observed at the bud neck or the presumptive bud sites. This localization is consistent with the data indicating that YlBud8 is not involved in the regulation of bipolar budding. Besides, Bud8 homolog proteins from other species also have different functions in different biological processes. For example, in Cryptococcus neoformans, BUD8 was significantly up-regulated in the $\mathrm{H} 99$ strain, which demonstrates higher virulence than wild-type strains, but its function in cell morphology requires further investigation [28].

\section{The Coiled-Coil Domain Is Essential for the Function of YlBud8}

The coiled-coil domain is an abundant structural motif that is present in approximately $10 \%$ of all proteins of any given species [29]. The amino acids in this domain can 
interact among themselves to form amphiphilic $\alpha$-helices, which then form a super-helix, i.e. the coiled-coil [30]. Coiled-coil domains have been visualized as rod-like spacers separating functional domains and they always act as protein scaffolds to mediate interactions between proteins [31, 32]. A coiled-coil domain exists at the $\mathrm{N}$ terminus of YlBud8 and we hypothesize that this domain can mediate the interaction between YlBud8 and other proteins to enable YlBud8 to perform its normal functions. To test this, truncated fragments of YlBud8 were constructed. Our results show that the fragments Ylbud8-1 and Ylbud8-3, which contain the coiled-coil domain but not the long N-terminus of YlBud8, can localize to the correct cellular sites, such as the cell membrane and the small bud cortex and perform their normal functions, as they are able to complement the cellular defects observed in the Ylbud8s mutant. However, when the coiled-coil domain was removed, as with Ylbud8-2 and Ylbud8-4 fragments, the fragments were neither able to perform normal functions nor localize to normal sites. These results indicate that it is the coiled-coil domain, but not the long N-terminus that plays important roles in the normal function of YlBud8. However, whether downstream and upstream signaling proteins of YlBud8 can act on YlBud8 through the coiledcoil domain has not been confirmed and further investigations will be required.

To date, no other homologs of Bud8 from organisms other than S. cerevisiae and C. neoformans have been reported, and our study will enrich the known functional diversity of Bud8 homologs. There are still many questions at present on the function of these proteins and further tests are required to determine if YlBud8 can act on YlRsr1 to play effector roles in cell separation. The functions of other domains of YlBud8 also require further study.

\section{Acknowledgments}

We thank X-D Gao for his support, helpful discussions and providing plasmids and yeast strains. This work was supported by the National Natural Science Foundation of China (no. 31500056), Scientific Research Fund for Ph.D. of Jining Medical University (no. JY2015BS13) and Supporting Fund for Teachers' Research of Jining Medical University (no. JY2017KJ012).

\section{Conflict of Interest}

The authors have no financial conflicts of interest to declare.

\section{References}

1. Martin SG, Chang F. 2003. Cell polarity: a new $\bmod (\mathrm{e})$ of anchoring. Curr. Biol. 13: R711-713.

2. Drubin DG, Nelson WJ. 1996. Origins of cell polarity. Cell 84: 335-344.

3. Schenkman LR, Caruso C, Pagé N, Pringle JR. 2002. The role of cell cycle regulated expression in the localization of spatial landmark proteins in yeast. J. Cell Biol. 156: 829-841.

4. Bi E, Park HO. 2012. Cell polarization and cytokinesis in budding yeast. Genetics 191: 347-387.

5. Pollard TD. 2010. Mechanics of cytokinesis in eukaryotes. Curr. Opin. Cell Biol. 22: 50-56.

6. Barr FA, Gruneberg U. 2007. Cytokinesis: placing and making the final cut. Cell 131: 847-860.

7. Chant J. 1999. Cell polarity in yeast. Annu. Rev. Cell Dev. Biol. 15: 365-391.

8. Li N, Michael S. 2001. A genomic study of the bipolar bud site selection pattern in Saccharomyces cerevisiae. Mol. Bio. Cell 12: 2147-2170.

9. Park HO, Bi E. 2007. Central roles of small GTPases in the development of cell polarity in yeast and beyond. Microbiol. Mol. Biol. Rev. 71: 48-96.

10. Park HO, Bi E, Pringle JR, Herskowitz I. 1997. Two active states of the Ras-related Bud1/Rsr1 protein bind to different effectors to determine yeast cell polarity. Proc. Natl. Acad. Sci. USA 94: 4463-4468.

11. Kang PJ, Béven L, Hariharan S, Park HO. 2010. The Rsr1/ Bud1 GTPase interacts with itself and the Cdc42 GTPase during bud-site selection and polarity establishment in budding yeast. Mol. Biol. Cell 21: 3007-3016.

12. Lee ME, Lo WC, Miller KE, Chou CS, Park HO. 2015. Regulation of Cdc42 polarization by the Rsr1 GTPase and Rga1, a Cdc42 GTPase-activating protein, in budding yeast. J. Cell Sci. 128: 2106-2117.

13. Basu S, Vadaie N, Prabhakar A, Li B, Adhikari H, Pitoniak A, et al. 2016. Spatial landmarks regulate a Cdc42-dependent MAPK pathway to control differentiation and the response to positional compromise. Proc. Natl. Acad. Sci. USA 113: 2019-2028.

14. Herrero AB, Lopez MC, Fernandez-Lago L, Dominguez A. 1999. Candida albicans and Yarrowia lipolytica as alternative models for analysing budding patterns and germ tube formation in dimorphic fungi. Microbiology 145: 2727-2737.

15. Gimeno CJ, Ljungdahl PO, Styles CA, Fink GR. 1992. Unipolar cell divisions in the yeast $S$. cerevisiae lead to filamentous growth: regulation by starvation and RAS. Cell 68: 1077-1090.

16. Veses VN, Gow NA. 2008. Pseudohypha budding patetrns of Candida albicans. Med. Mycol. 47: 268-275.

17. Nicaud JM. 2012. Yarrowia lipolytica. Yeast 29: 409-418.

18. Kang PJ, Angerman E, Nakashima K, Pringle JR, Park, HO. 2004. Interactions among Rax1p, Rax2p, Bud8p, and Bud9p 
in marking cortical sites for bipolar bud-site selection in yeast. Mol. Biol. Cell 15: 5145-5157.

19. Kato Y, Kawasaki H, Ohyama Y, Morishita T, Iwasaki H, Kokubo T, et al. 2011. Cell polarity in Saccharomyces cerevisiae depends on proper localization of the Bud9 landmark protein by the EKC/KEOPS complex. Genetics 188: 871-882.

20. Harkins HA, Page N, Schenkman LR, Virgilio DV, Shaw S, Bussey $\mathrm{H}$, et al. 2001. Bud8 and Bud9, proteins that may mark the sites for bipolar budding in yeast. Mol. Bio. Cell 12: $2497-2518$.

21. Li YQ, Li M, Zhao XF, Gao XD. 2014. A role for the Rap GTPase YIRsr1 in cellular morphogenesis and the involvement of YIRsr1 and the Ras GTPase YlRas2 in bud site selection in the dimorphic yeast Yarrowia lipolytica. Eukaryot. Cell 13: 580-590.

22. Richard M, Quijano RR, Bezzate S, Bordon-Pallier F, Gaillardin C. 2001. Tagging morphogenetic genes by insertional mutagenesis in the yeast Yarrowia lipolytica. J. Bacteriol. 183: 3098-3107.

23. Zhao XF, Li M, Li YQ, Chen XD, Gao XD. 2013. The TEA/ ATTS transcription factor YlTec1p represses the yeast-hypha transition in the dimorphic yeast Yarrowia lipolytica. FEMS Yeast Res. 13: 50-61.

24. Li M, Li YQ, Zhao XF, Gao XD. 2014. Roles of the three Ras proteins in the regulation of dimorphic transition in the yeast Yarrowia lipolytica. FEMS Yeast Res. 14: 451-463.

25. Lupas A. 1996. Coiled coils: new structures and new functions. Trends Biochem. Sci. 21: 375-382.

26. Taheri N, Kohler T, Braus GH, Mosch HU. 2000. Asymmetrically localized Bud8p and Bud9p proteins control yeast cell polarity and development. EMBO J. 19: 6686-6696.

27. Krappmann AB, Taheri N, Heinrich M, Mosch HU. 2007. Distinct domains of yeast cortical tag proteins Bud8p and Bud9p confer polar localization and functionality. Mol. Biol. Cell. 18: 3323-3339.

28. Movahed E, Munusamy K, Tan GM, Looi CY, Tay ST, Wong WF. 2015. Genome-wide transcription study of Cryptococcus neoformans H99 clinical strain versus environmental strains. PLoS One 10: $\mathrm{e} 0137457$.

29. Liu J, Rost B. 2001. Comparing function and structure between entire proteomes. Protein Sci. 10: 1970-1979.

30. Surkont J, Pereira-Leal JB. 2015. Evolutionary patterns in coiled-coils. Genome Biol. Evol. 7: 545-556.

31. Zhang H, Chen J, Wang Y, Peng L, Dong X, Lu Y, et al. 2009. A computationally guided protein-interaction screen uncovers coiled-coil interactions involved in vesicular trafficking. J. Mol. Biol. 392: 228-241.

32. Munro S. 2011. The golgin coiled-coil proteins of the Golgi apparatus. Cold Spring Harb. Perspect. Biol. 3: 1-14. 